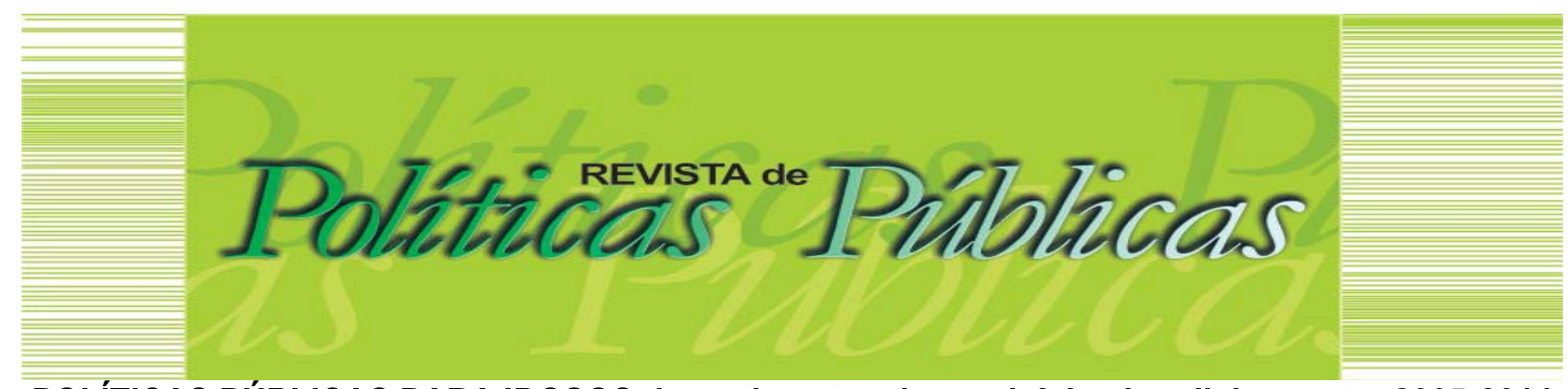

\title{
POLÍTICAS PÚBLICAS PARA IDOSOS: investimentos de municípios brasileiros entre 2005-2014
}

\author{
Marcelo de Maio Nascimento 1 \\ Carolina Nascimento Silva ${ }^{2}$ \\ Danielly Elizeu Alves ${ }^{3}$ \\ Edmilson Santos dos Santos 4
}

\begin{abstract}
Resumo
Este estudo tem como objetivo avaliar os níveis de adesão dos municípios brasileiros das cinco regiões do território nacional à função Assistência Social (FAS) e à subfunção Assistência ao Idoso (SAI), entre 2005 a 2014. Verifica que 0 índice de adesão dos municípios à FAS e SAI foi de $90 \%$ e $50 \%$, respectivamente. Municípios localizados em estados com baixo Índice de Desenvolvimento Humano na região Norte e Nordeste apontaram menor adesão à SAl, indicando que desigualdades socioeconômicas impedem a participação em políticas públicas da federação. Conclui que as estratégias de municipalização da política de assistência social ao idoso (2005-2014) seguiram as diretrizes de agendas internacionais e nacionais relativas ao envelhecimento saudável. Os achados podem preencher lacunas da literatura brasileira na área de políticas públicas.
\end{abstract}

Palavras-chave: Política pública. Envelhecimento. Idoso. Assistência. Saúde.

\section{PUBLIC POLICIES FOR THE ELDERLY: investments by Brazilian municipalities between 2005-2014}

\begin{abstract}
This study aimed to evaluate the levels of adhesion of Brazilian municipalities in the five regions of the national territory to the Social Assistance function (FAS) and to the sub-function Assistance to the Elderly (SAI), between 2005 and 2014. It was found that the adhesion index of municipalities to FAS and SAI was $90 \%$ and $50 \%$, respectively. Municipalities located in states with a low Human Development Index in the North and Northeast regions showed lower adherence to SAI, indicating that socioeconomic inequalities prevent participation in public policies of the federation. It was concluded that the municipalization strategies of the social assistance policy for older adults (2005-2014) followed the guidelines of international and national agendas related to healthy aging. The findings can fill gaps in Brazilian literature in the area of public policy.

Keywords: Public policy. Aging. Elderly. Assistance. Health.
\end{abstract}

Artigo recebido em: 31/05/2021 Aprovado em: 30/11/2021

DOI: http://dx.doi.org/10.18764/2178-2865.v25n2p937-949

\footnotetext{
1 Professor do curso de Educação Física da Universidade Federal do Vale do São Francisco. E- mail: Marcelo.nascimento@univasf.edu.br

2 Graduanda em Psicologia da Universidade Federal do Vale do São Francisco, membro da Universidade Aberta à Terceira Idade. E-MAIL: carolina.cns05@gmail.com

3 Graduanda em Enfermagem da Universidade Federal do Vale do São Francisco, membro da Universidade Aberta à Terceira Idade. E-mail: daniellyelizeualves@gmail.com

4 Professor do curso de Educação Física da Universidade Federal do Vale do São Francisco. E-mail: edmilson.santos@univasf.edu.br
} 


\section{INTRODUÇÃO}

Há três décadas a Organização Mundial de Saúde advertiu sobre as consequências do processo do envelhecimento humano para as populações e seus governantes (WHO, 1992). Por essa razão, foram sugeridas propostas estruturais à criação de políticas públicas para que se pudesse aumentar os investimentos à atenção do idoso nas áreas da educação, saúde, direitos humanos, cultura e lazer (WHO, 2002, 2014, 2015). Diante disso, não é por acaso, que o envelhecimento foi considerado como um dos fenômenos mais relevantes do século XXI (VERAS, 2009).

O rápido e progressivo aumento do número de idosos é um reflexo da queda das taxas de natalidade, seguido por avanços promovidos nas condições de saúde da população. Muitos países enfrentam problemas por causa da transição demográfica, caracterizada por alterações significativas na composição das faixas etárias de uma população (WONG; CARVALHO, 2006; IBGE, 2017). No caso do Brasil, conforme estimativas do Instituto Brasileiro de Geografia e Estatística (IBGE, 2017),no ano de 1940 , brasileiros viviam em média 45,5 anos, no ano de 2017 , a taxa subiu para 76,6 anos. A estimativa para o ano de 2030 é de que o Brasil apresente a quinta maior população idosa do planeta e no ano de2050, 25\% da população terão idade igual ou superior a 80 anos (IBGE, 2019). Portanto, um número considerável de brasileiros com necessidade de cuidados especiais (LEVY et al., 2018).

A implementação de políticas para a população idosa depende da alocação de recursos. Ainda hoje, pouco se sabe sobre os investimentosdos entes federados (União, Unidades da Federação e municípios) em políticas específicas para a população idosa. Em 1999 foi criada a portaria ministerial de número 42 do então Ministério do Orçamento e Gestão, que permite acompanhar os gastos públicos nas diferentes funções sociais dosgovernos. Atualmente, as funções são em número de 28, que, por conseguinte, possuem subfunções, cujo papel é sumarizar os serviços públicos relacionados a um tema específico. A função de número 8 é intitulada como "Assistência Social", composta por quatro subfunções, cada uma identificada com seu respectivo código: 241 Assistência ao Idoso, 242 Assistência ao Portador de Deficiência, 243 Assistência à Criança e ao Adolescente e 244 Assistência Comunitária. Este sistema de classificação numérico é padrão, agilizando os procedimentos do orçamento tanto da União, como dos 26 estados, do Distrito Federal e dos 5.570 municípios.

Considerando a escassez de informações (dados fiscais) sobre o comportamento das verbasalocadas pelos municípios em ações direcionadas à população idosa, o presente estudo teve como objetivo avaliar os níveis de adesão dos municípios brasileiros na função "Assistência Social" (FAS) e na subfunção "Assistência ao Idoso" (SAI). 


\section{ENVELHECIMENTO HUMANO E GESTÃO PÚBLICA}

Os reflexos do envelhecimento humano não são inexpressivos, pois podem conduzir a gestão pública ao colapso (DALLMEYER; WICKER; BREUER, 2017). Miranda et al. (2016) destacaram que a sociedade deve estar consciente que o envelhecimento associado à carência de suporte básico representa que, provavelmente no futuro, todos os cidadãos irão arcar com altos custos de investimento social. Para evitar o repasse das despesas à população é necessário que o Estado crie políticas, mas, que elas sejam fiscalizadas. Essa medida aumenta as chances para que idosos recebam a atenção e o cuidado de que necessitam e possam desfrutar de um envelhecimento com qualidade (WONG; CARVALHO, 2006).

Willig, Lenardt e Méier (2012) destacaram que a criação de políticas específicas para a população brasileira permitiu conquistas consideráveis na área do direito do idoso. Contudo, ainda seria necessário o desenvolvimento de estudos transversais e longitudinais para analisar o emprego dos repasses a nível setorial: municípios.Um dos principais marcos de proteção ao idoso ocorreu com a criação da Lei 8.842/94, que dispõe sobre a Política Nacional do Idoso (PNI) (Ministério da Saúde, 1994), estabelecendo oConselho Nacional do Idoso. Dois outros artifícios foram a Política Nacional de Saúde à Pessoa Idosa (PNSPI) (Ministério da Saúde, 1999) e o Estatuto do Idoso (Ministério da Saúde, 2003). Essas políticas contribuíram significativamente para a descentralização das funções sociais, propiciando avanços no enfrentamento das exigências geradas pelo envelhecimento, além de terem regulamentado,entre a família e o Estado, a divisão da responsabilidade sobre a assistência ao idoso (VERAS; OLIVEIRA, 2018).

No Brasil, diferentemente dos países desenvolvidos, o envelhecimento populacional vem ocorrendo em um cenário socioeconômico recessivo, logo, desfavorável à consolidação de um sistema de proteção social sustentável (CAMARANO; PASINATO, 2004). Entre os problemas existentes para que se atinja um envelhecimento igualitário há a desigualdade na distribuição dos recursos da Federação para os estados (ARAÚJO; FLORES, 2017). Em consequência disso, surgem falhas nos mecanismos responsáveis pela distribuição de verbas às regiões, que são fortemente caracterizadas por desigualdades. Outra questão incide na estrutura fiscal adotada, que geralmente não contribui para ações duradouras, visto que as medidas acabam desprivilegiando um ou outro estado da Federação.

Como ente federado, os municípios possuem relativa autonomia fiscal e política que thes permite definir as políticas de investimento. No entanto, fatores como diferenças fiscais, bem como a capacidade para arrecadar e gastar fundos variam entre os municípios brasileiros, gerando desigualdades à locação das verbas (GOMES; DOWELL, 2000; SOUZA, 2001; AFONFO; LOBO, 2002; AFONSO, 2003). A Constituição de 1988 conferiu autonomia político administrativa aos municípios, que 
passaram a ser entes da federação com autonomia para formular e implementar políticas próprias. № entanto, o fato trouxe um desafio para a gestão pública, que foi a coordenação intergovernamental. De forma geral, a descentralização é um processo político e não técnico-administrativo (SILVEIRA, ROCHA; COSTA, 2005), sua proposta se insere no contexto da redemocratização, uma vez que busca restaurar a Federação, majorando o poder político e tributário das entidades subnacionais. Como resultado, há o empoderamento das comunidades, algo útil à consolidação do processo decisório das políticas públicas.

Conforme Arretche (2010), a descentralização de políticas públicas exige a distinção conceitual entre a responsabilidade pela execução de políticas públicas (policy-making) e a autoridade para tomar decisões sobre essas políticas (policydecision-making). Logo, no contexto da promoção da saúde do idoso, a consideração desses fatores é determinante porque implica diretamente sobre a arrecadação dos valores da FAS e, por conseguinte, sobre a redistribuição dos fundos nas quatro subfunções ${ }^{1}$. Conforme Arretche (2010, p. 596-597):

As decisões de arrecadação tributária e de gasto dos governos subnacionais - tanto de
estados quanto de munííios - são significativamente limitadas pela legislação nacional.
Além disto, a provisão de serviços públicos e a alocação setorial do gasto são fortemente
afetadas pela legislação e supervisão federais. Como consequência, embora as unidades
constituintes sejam politicamente autônomas e tenham responsabilidade na arrecadação de
tributos e de execução de políticas, sua autonomia decisória não pode ser adequadamente
interpretada, se ignorarmos a extensão em que a agenda dos governos subnacionais é
afetada pela regulação federal.

Há três décadas Almeida (1995) informou que apesar do modelo federalista brasileiro não ter definido funções claras entre os entes federados em muitos pontos da agenda social, os municípios pressionados por demandas locais ou por decisões da elite política poderiam estar realizando alocações importantes da agenda social, o que inclui a assistência ao idoso. Segundo Berzins, Giacomin e Camarano (2016), os investimentos na área da Assistência Social ao Idoso cresceram ao longo dos anos. Entretanto, um enigma permaneceu desconhecido, que é se o compromisso assumido pelos responsáveis por essas políticas sobrevém da realidade (aumento da população idosa) ou se é uma resposta às demandas e à pressão das partes interessadas (stakeholders).

\section{MÉTODOS}

Trata-se de um estudo descritivo que analisou o comportamento dos "decisores" locais(prefeitos)sobre a adesão às políticas públicas na área da pessoa idosa. Os objetivos específicos foram: i) identificar o comportamento dos "decisores" dos municípios brasileiros ao longo do período do 
estudo; ii) examinar a taxa de adesão dos municípios brasileiros segundo as cinco regiões do território nacional à função "Assistência Social" e à subfunção "Assistência ao Idoso".

Esta política provocou importantes mudanças no contexto da gestão e operacionalização da Política de Assistência Social no Brasil (NOB/SUAS, 2005; 2012). Os indicadores utilizados foram "Assistência Social" e "Assistência ao Idoso" O conjunto dos dados utilizados são originários do domínio público, disponibilizados no site do Tesouro Nacional (https://siconfi.tesouro.gov.br/siconfi/pages/publi) referentes às contas anuais apresentadas no Sistema de Informações Contábeis e Fiscais do Setor Público Brasileiro- Siconfi. Foi elaborada planilha de dados, em que constam os 5.570 municípios, sua distribuição regional e estadual e adesão à função "Assistência Social" e à subfunção "Assistência ao Idoso". Para tratamento, utilizou-se a estatística descritiva. 0 acesso aos dados foi realizado entre os dias 15-20 de maio de 2019.

\section{RESULTADOS}

A Tabela 1 apresenta resultados percentuais sobre a adesão dos 5.570 municípios brasileiros à função Assistência Social e à subfunção Assistência ao Idoso, no período de 2005 a 2014. Verificou-se que 5.256 dos municípios aderiram à FAS e 2.750 à SAI. Salienta-se que os coeficientes da SAI são tendencialmente menores que os da FAS, visto que sua adesão é optativa, pois são os municípios que decidemem contemplar ou não uma ou outra agenda proposta pelo Governo. Ademais, a função Assistência Social possui outras três subfunções além da Assistência ao Idoso, que poderiam estar sendo contempladas.

$\mathrm{Na}$ Tabela1, verifica-se que a adesão à FAZ se manteve constante na casa dos $90 \%$, durante dez anos, o queindica excelente envolvimento dos municípios com esta agenda. No ano de 2007, a taxa de adesão chegou a 98,04\%. Paralelo ao fato, observa-se que a adesão dos municípios brasileiros à SAI variou de 43,15 a 53,51\%. Para uma agenda em que não há obrigação de investimentos, ospercentuais encontrados são significativos, atestando para o fato de que os municípios brasileiros estão atentos, ou preocupados, com a transição da pirâmide etária.

Tabela 1 - Adesão de todos os municípios brasileiros à Função Assistência Social e SubfunçãoAssistência ao Idoso, 2005 a 2014.

\begin{tabular}{|c|c|c|c|c|}
\hline Período & AdesãoFAS & $\%$ & AdesãoSAI & $\%$ \\
\hline 2005 & 5205 & 93,44 & 2404 & 43,15 \\
\hline 2006 & 5077 & 91,14 & 2714 & 48,72 \\
\hline 2007 & 5461 & 98,04 & 2664 & 47,82 \\
\hline 2008 & 5336 & 95,79 & 2664 & 47,82 \\
\hline 2009 & 5366 & 96,33 & 2803 & 50,32 \\
\hline 2010 & 5332 & 95,72 & 2944 & 52,85 \\
\hline
\end{tabular}




\begin{tabular}{|l|l|l|l|l|}
\hline 2011 & 5322 & 95,54 & 2981 & 53,51 \\
\hline 2012 & 5114 & 91,81 & 2760 & 49,55 \\
\hline 2013 & 5315 & 95,42 & 2928 & 52,56 \\
\hline 2014 & 5129 & 92,08 & 2639 & 47,37 \\
\hline
\end{tabular}

Fonte: Elaborado pelo autor, 2021.

Legenda: FAS: Função Assistência Social; SAl: Subfunção Assistência ao idoso.

Os dados agregados nacionalmente não permitem, diante das desigualdades fiscais dos municípios brasileiros, compreender o impacto da região na adesão à agenda. Elas diferem tanto em número de municípios como em capacidade fiscal. Apesar da região Norte ser formada por apenas 450 municípios (Tabela 2), é possível observar que foi alto o percentual de adesão dos municípios à agenda FAS no período de 2005 a 2014. Destaca-se, todavia, considerável oscilação do índice de adesão dos municípios da região Norte à FAS entre os anos de 2005 e 2012.

Traçando um paralelo entre os resultados da Tabela 2 com a Tabela 1, pode-se observar que os municípios da região Norte apresentaram, para todo o período, percentual de adesão ao FAS compatível com as taxas de adesão nacional. Por outro lado, comparativamente, a adesão à SAI se apresentou por vezes abaixo do índice nacional, o que pode ser observado nos anos de 2005, 20072008, 2011-2012 e 2014. Diante do impacto futuro da população idosa, é necessário criar mecanismos de correção deste comportamento.

Tabela 2 - Adesão de municípios do Norte à Função Assistência Social e Subfunção Assistência ao Idoso, 2005 a 2014.

\begin{tabular}{|c|c|c|c|c|}
\hline Período & AdesãoFAS & $\%$ & Adesão SAl & $\%$ \\
\hline 2005 & 395 & 87,77 & 167 & 37,11 \\
\hline 2006 & 442 & 98,22 & 194 & 43,11 \\
\hline 2007 & 433 & 96,22 & 174 & 38,66 \\
\hline 2008 & 423 & 94,00 & 167 & 37,11 \\
\hline 2009 & 433 & 96,22 & 188 & 41,77 \\
\hline 2010 & 430 & 95,55 & 197 & 43,77 \\
\hline 2011 & 407 & 90,44 & 178 & 39,55 \\
\hline 2012 & 373 & 82,88 & 163 & 36,22 \\
\hline 2013 & 406 & 90,22 & 190 & 42,22 \\
\hline 2014 & 408 & 90,66 & 178 & 39.55 \\
\hline
\end{tabular}

Fonte: Elaborado pelo autor, 2021.

Legenda: FAS: Função Assistência Social; SAl: Subfunção Assistência ao idoso.

Entre as cinco regiões do território nacional, o Nordeste é a que apresenta o maior número de municípios:1.794 unidades (Tabela 3). A análise mostrou que proporcionalmente 1.676 dos municípios aderiram à FAS, enquanto 725 municípios investiram recursos em agendas da SAI.O percentual da adesão à SAl oscilou consideravelmente no ano de 2009, que foi um marco divisor, pois até 2008 a taxa de adesão era de $30 \%$, aumentando para $40 \%$ ou mais a partir de 2009 . 
Quando comparamos os resultados da Tabela 3 com a Tabela 1, verifica-se equilíbrio dos municípios da região Nordeste entre sua adesão e a adesão à FAS com o índice nacional. Em relação à SAI, quando comparada ao índice nacional, verificou-se compatibilidade entre os resultados da região Nordeste com a região Norte, visto que existiu baixa adesão à SAl de 2005 a 2008 e no ano de 2014.

Tabela 3 - Adesão de municípios do Nordeste à Função Assistência Social e Subfunção Assistência ao idoso, 2005 a 2014.

\begin{tabular}{|c|c|c|c|c|}
\hline Período & Adesão FAS & $\%$ & Adesão SAI & $\%$ \\
\hline 2005 & 1598 & 89,07 & 601 & 33,50 \\
\hline 2006 & 1736 & 96,76 & 695 & 38,74 \\
\hline 2007 & 1723 & 96,04 & 692 & 38,57 \\
\hline 2008 & 1706 & 95,09 & 698 & 38,90 \\
\hline 2009 & 1735 & 96,71 & 745 & 41,52 \\
\hline 2010 & 1721 & 95,93 & 805 & 44,87 \\
\hline 2011 & 1660 & 92,53 & 783 & 43,64 \\
\hline 2012 & 1537 & 85,67 & 721 & 40,18 \\
\hline 2013 & 1680 & 93,64 & 801 & 46,64 \\
\hline 2014 & 1663 & 92,69 & 713 & 39,74 \\
\hline
\end{tabular}

Fonte: Elaborado pelo autor, 2021

Legenda: FAS: Função Assistência Social; SAI: Subfunção Assistência ao idoso.

A Tabela 4 apresenta dados referentes aos 466 municípios da região Centro-Oeste. Em se tratando da taxa de adesãoà FAS, 414 dos municípios aderiram a esta agenda e 262 à SAI. A análise revelou que a participação dos municípios àFAS entre os anos 2005-2007 e 2011-2014 foi superior a $88 \%$, enquanto que no período de $2008-2010$, o percentual reduziu consideravelmente para $070 \%$. Seguindo a ordem dos resultados nacionais, a taxa de adesão à SAI foi semelhante às demais regiões, visto que ficou entre 50,21-59,87\%.

A comparação do percentual de adesão àFAS pelos municípios da região Centro-Oeste com os índices nacionais (Tabela 1) revelou $20 \%$ de baixa na aderência a essa agenda entre os anos de 2008-2010, mantendo-se, contudo, concisa ao índice nacional nos demais anos do período analisado. No que diz respeito à SAI, a região Centro-Oeste indicou percentuais de adesão significativos para todo o período analisado. Os resultados foram inclusive superiores à taxa nacional entre os anos de 2005-2008, bem como, entre 2012 e 2014

Tabela 4 - Adesão de municípios do Centro-Oeste à Função Assistência Social e SubfunçãoAssistência ao Idoso, 2005 a 2014.

\begin{tabular}{|c|c|c|c|c|}
\hline Período & Adesão FAS & $\%$ & Adesão SAI & $\%$ \\
\hline 2005 & 435 & 93,34 & 234 & 50,21 \\
\hline 2006 & 465 & 99,78 & 263 & 56,43 \\
\hline 2007 & 460 & 98,71 & 264 & 56,65 \\
\hline 2008 & 358 & 76,82 & 254 & 54,50 \\
\hline 2009 & 356 & 76,39 & 279 & 59,87 \\
\hline 2010 & 351 & 75,32 & 259 & 55,57 \\
\hline 2011 & 442 & 94,84 & 271 & 58,15 \\
\hline 2012 & 434 & 93,13 & 266 & 57,08 \\
\hline
\end{tabular}




\begin{tabular}{|l|l|l|l|l|}
\hline 2013 & 427 & 91,63 & 278 & 59,65 \\
\hline 2014 & 413 & 88,62 & 253 & 54,29 \\
\hline
\end{tabular}

Fonte: Elaborado pelo autor, 2021.

Legenda: FAS: Função Assistência Social; SAl: Subfunção Assistência ao idoso.

Com base na Tabela 5, verifica-se que o quantitativo de participação dos 1.668 municípiosda região Sudeste na funçãoFAS foi de 1.590 e 897nasubfunção SAI. Com exceção do ano de 2006 existiu alto nível de adesão dos municípios à agenda FAS, a taxa de adesão desses municípios para o período de 2005-2014 foi em média de 90\%. Em relação à SAl, observou-se que os coeficientes de adesão permaneceram constantes, entre 51,25 a $59,41 \%$, em um período de nove anos.

Apenas no ano de 2005 a adesão dos municípios à SAl foi baixa, ou seja: 43,3\%. Na comparação entre os resultados da Tabela 5 e Tabela 1, verificou-se que a adesão à FAS, com exceção do ano de 2006, manteve-se nos padrões nacionais com índices de $90 \%$. Em relação à SAI os valores foram semelhantes à média da taxa nacional e compatíveis com a região Centro-Oeste e superiores às regiões Norte e Nordeste.

Tabela 5-Adesão de municípios do Sudesteà Função Assistência Social e SubfunçãoAssistência ao Idoso, 2005 a 2014.

\begin{tabular}{|c|c|c|c|l|}
\hline Período & Adesão FAS & $\%$ & Adesão SAI & $\%$ \\
\hline 2005 & 1605 & 96,22 & 723 & 43,34 \\
\hline 2006 & 1248 & 74,82 & 855 & 51,25 \\
\hline 2007 & 1661 & 99,58 & 860 & 51,55 \\
\hline 2008 & 1664 & 99,76 & 861 & 51,61 \\
\hline 2009 & 1657 & 99,34 & 910 & 54,55 \\
\hline 2010 & 1653 & 99,10 & 971 & 58,21 \\
\hline 2011 & 1634 & 97,96 & 991 & 59,41 \\
\hline 2012 & 1591 & 95,38 & 924 & 55,39 \\
\hline 2013 & 1625 & 97,42 & 969 & 58,09 \\
\hline 2014 & 1564 & 93,76 & 906 & 54,31 \\
\hline
\end{tabular}

Fonte: Elaborado pelo autor, 2021

Legenda: FAS: Função Assistência Social; SAI: Subfunção Assistência ao idoso.

A região Sul do Brasil possui 1.191 municípios (Tabela 6). Desses, 1.170 aderiram à FAS e 679 à SAI. De forma geral, a participação desses municípios à FAS foi alta e constante ao longo de dez anos. Por conseguinte, o percentual de participação dos municípios da região Sul na agenda SAI foi de $50 \%$.

Quando comparamos o percentual de adesão à FAS dos municípios da região Sul com os índices nacionais, observamos taxas significativamente altas, próximas a 100\%. Em relação à adesão dos municípios sulistas à SAI, verificou-se compatibilidade com os índices nacionais (Tabela 1), uma 
vez que os valores atestaram para casa dos 50\%, com exceção do ano de 2014, que foi levemente inferior: $49,45 \%$.

Tabela 6 - Adesão de municípios do Sul Função Assistência Social e Subfunção Assistência ao Idoso, 2005 a 2014.

\begin{tabular}{|c|c|c|c|c|}
\hline Período & Adesão FAS & $\%$ & Adesão SAl & $\%$ \\
\hline 2005 & 1172 & 98,40 & 679 & 57,01 \\
\hline 2006 & 1186 & 99,58 & 707 & 59,36 \\
\hline 2007 & 1184 & 99,41 & 674 & 56,59 \\
\hline 2008 & 1185 & 99,49 & 684 & 57,43 \\
\hline 2009 & 1185 & 99,49 & 681 & 57,17 \\
\hline 2010 & 1177 & 98,82 & 712 & 59,78 \\
\hline 2011 & 1179 & 98,99 & 695 & 58,35 \\
\hline 2012 & 1179 & 98,99 & 686 & 57,59 \\
\hline 2013 & 1177 & 98,82 & 690 & 57,93 \\
\hline 2014 & 1081 & 90,76 & 589 & 49,45 \\
\hline
\end{tabular}

Fonte: Elaborado pelo autor, 2021

Legenda: FAS: Função Assistência Social; SAl: Subfunção Assistência ao idoso.

\section{DISCUSSÃO}

A análise dos dados sobre a adesão dos 5.265,7 municípios das cinco regiões do território nacionalmostrou níveis consideráveis de adesão à $\mathrm{FA}, 75,32-99,76 \%$, e moderados em relação à SAI, 33,50-59,65\%. Considerando a função Assistência Social possuir além da subfunção Assistência ao Idoso, outras quatro subfunções (242 Assistência ao Portador de Deficiência, 243 Assistência à Criança e ao Adolescente e 243 Assistência Comunitária), pode-se dizerque, ao longo de dez anos, os valores verificados para SAI se mostraram constantes. Isso indica que os municípios brasileiros se comprometeramcom as diretrizes de agendas nacionais e internacionais do cuidado e atenção ao idoso.

Em se tratando da análise setorial, verificou-se que a taxa de adesão à FAS e à SAI se mostrou diferente entre as regiões. A causa identificada foi o nível de desenvolvimento das regiões. Conforme 0 Instituto de Pesquisa Econômica Aplicada (IPEA, 2016) as regiões do país com maior Índice de Desenvolvimento Humano (IDH), como o Sul, Sudeste e o Centro-Oesteapresentam maior expectativa de vida. Essas regiões também possuem um quantitativo elevado de indivíduos em idade igual ou superior a 60 anos, acompanhado por um baixo coeficiente de cidadãos em situação de vulnerabilidade. Assim, os achados do presente estudo corroboraram com dados do IPEA (2016), pois, comparativamente, verificou-se que municípios das regiões Norte e Nordeste apresentaram baixa índicede adesão à SAI. Sabe-se que essas regiões apresentam níveis de desenvolvimento econômico 
e social mais baixos e, por conseguinte, maior chance de desigualdades sociais (AFONSO, 2003; GOMES; DOWELL, 2000). Esse resultado pode ser interpretado como necessidade para queo Governo Federal coordene melhor as políticas de assistência ao idoso, reduzindo assimetrias (ARRETCHE, 2010).

Outro ponto a considerar consiste na possibilidade de quealguns municípios tenham desenvolvido atividades com a população idosa, entretanto, por questões desconhecidas, os responsáveis tenham indicado no sistema outras subfunções relacionadas à pessoa idosa, como, por exemplo: esporte, lazer ou educação. 0 fato pode ser assumido como uma limitação do presente estudo e não exclui a possibilidade de que municípios localizados emregiõesde vulnerabilidadetenhamdesenvolvido políticas de apoio aos idosos, entre os anos de 2005-2014.

Nesse contexto, deve-se considerar que a taxa de adesão à SAI pode ter sido influenciada pela própria instância dos municípios(CAMARANO; PASINATO, 2004). Pois, com a reforma fiscal promovida pela Constituição Federal de 1988, a Federação possibilitou as entidades federadas implementarem políticas públicas de forma autônoma. Diante disso, tanto a União, como os estados,incluindo oDistrito Federal e os municípios desfrutam do livre arbítrio para realizar investimentos(ANDRADE et al., 2013).Isso significa dizer que, como entes federados, os municípios possuem autoridade política para fazer escolhas, deliberando suas preferências para alocação dos recursos do orçamento.

A questão suscita reflexões, pois a autonomia fiscal ao invés de produzir um cenário de maior responsabilidade dos entes municipais, pode aumentar as desigualdades sociais e políticas (WILLIG; LENARDT; MÉIER, 2012). Sendo assim, os arranjos adotados pelos municípios em sua estrutura de financiamento definem o grau de prioridade nas políticas.A literatura federalista aponta com muita precisão que a autonomia a locativa produz diferenças na capacidade de escolha das agendas e que elas podem promover desigualdades (ARRETCHE, 2010). Sendo assim, a diferença observada sobre os índices de adesão da SAI pelos municípios das regiões Norte e Nordeste,em relação à taxa nacional, pode ser considerada como exemplo da fragilidade fiscal.É importante salientar que momentos de conjuntura recessiva são acompanhados por crise fiscal, o que aumentam as chances de cortes à expansão do sistema de proteção socialpara todos os grupos etários (CAMARANO; PASINATO, 2004).

Os resultados do presente estudo também permitiram constatar que a maioria dos municípios brasileiros se mantiveram comprometidos com agendas criadas para a população idosa, durante dez anos. $O$ achado abre espaço para aprofundar análises, como por exemplo, compreender se a receita corrente, a receita própria ou as receitas de transferências constitucionais impactam na capacidade dos gastos dos municípios. Outra análise seria verificar se o percentual de idosos 
residentes nos municípios carece de mais investimentos e novas políticas, bem como, até que ponto 0 nível de desenvolvimento humano de uma região impacta a adesão dos municípios às agendas da área do envelhecimento humano.

\section{CONCLUSÃO}

Os resultados do presente estudo são reveladores, pois sugerem que no período de 20052014 a estratégia de municipalização da política de assistência social ao idoso foi bem-sucedida. Com base nos resultados, foi possível perceber que existiu alta adesão à FAS, com exceção dos municípios da região Centro-Oeste. Contudo, o fato foi observado apenas entre os anos de 2008-2010. Em relação à SAl, observou-se comprometimento das agendas setoriais com a populaçãoidosa, dado que sua adesão é voluntária.Essa informação é expressiva, pois indica que independente de questões partidárias, os governantes e os municípios não só criaram políticas públicas para a pessoa idosa, como deram continuidade às atividades das gestões anteriores. Isso demonstra comprometimento com as agendas das organizações internacionais, como é o caso da OMS.Por outro lado, a variação no comportamento dos municípios ao longo da série é compatível com a autonomia que lhes é outorgada na matéria.

O comprometimento dos gestores com demandas da área da assistência social mostrou seu interesse para consolidar políticasque estivessem em acordo com as diretrizes da Política Nacional de Saúde do Idoso. Isso implica a promoção do envelhecimento saudável, que diz respeito à melhoria, manutençãoou reabilitação da capacidade funcional dos idosos, a criação de medidas de prevenção de doenças ou à recuperação da saúde, com o objetivo de que os indivíduos se mantenham autônomos para realizarem as atividades de vida diária. Sendo assim, pode-se dizer que,entre os anos 2005-2014, os municípios investiram na mudança de paradigmas, o que na esfera municipal está relacionado à criação de parcerias e/ou à integração de setores públicos.

Os achados do presente estudo preenchem lacunas da literatura especializada tanto da área das políticas públicas, como da gerontologia, visto que pouco se conhece sobre o comportamento setorial (municípios) das políticas públicas do idoso. Em geral, os estudos se limitam a discutir o papel dos estados e, principalmente, da federação nas agendas. Nessa perspectiva,o presente estudo pode contribuir significativamente para a descentralização da pesquisa na área do envelhecimento humano e de políticas direcionadas a esta população.Diante desses resultados, outras questões se abrem como por exemplo: (a) qual o comportamento dos recursos alocados em função da capacidade fiscal de cada município; (b) em que medida o nível de desenvolvimento humano impacta na capacidade decisória; (c) 
se os recursos e sua evolução acompanham o peso da população idosa no orçamento; (d) e, do ponto de vista da policyanalysis, que fatores afetam o comportamento dos decisores.

\section{REFERÊNCIAS}

ANDRADE, L. M. et al. Políticas públicas para pessoas idosas no Brasil: Uma revisão integrativa.

Ciencia e Saude Coletiva, v. 18, n. 12, p. 3543-3552, 2013.

ARAÚJO, V.; FLORES, P. Redistribuição de renda, pobreza e desigualdade territorial no Brasil.

Revista de Sociologia e Politica, v. 25, n. 63, p. 159-182, 2017.

ARRETCHE, M. Federalismo e Igualdade Territorial: Uma Contradição em Termos? Dados, v. 53, n. 3, p. 587-620, 2010.

BERZINS, M. A. V. S.; GIACOMIN, K. C.; CAMARANO, A. A. A Assistência social na política nacional do idoso. 2016.

CAMARANO, A.; PASINATO, M. 0 envelhecimento populacional na agenda das políticas públicas. Os novos idosos brasileiros: muito além dos 60?, v. 60. p. 253-292, 2004.

DALLMEYER, S.; WICKER, P.; BREUER, C. How an aging society affects the economic costs of inactivity in Germany: empirical evidence and projections. European Review of Aging and Physical Activity, v. 14, n. 1, p. 1-9, 2017.

IBGE-INSTITUTO BRASILEIRO DE GEOGRAFIA E ESTATÍSTICA. IBGE lança estudo metodológico sobre mudança demográfica e projeções de população. Disponível em: https://agenciadenoticias.ibge.gov.br/agencia-sala-de-imprensa/2013-agencia-denoticias/releases/9831-ibge-lanca-estudo-metodologico-sobre-mudanca-demografica-e-projecoes-depopulacao. Acesso em: 20 jan. 2020.

IBGE-INSTITUTO BRASILEIRO DE GEOGRAFIA E ESTATÍSTICA. Projeção da População do Brasil por sexo e idade: 2000-2060. Disponível em:

https://ww2.ibge.gov.br/home/estatistica/populacao/projecao_da_populacao/2013/default.shtm.

IBGE-INSTITUTO BRASILEIRO DE GEOGRAFIA E ESTATÍSTICA.Projeção da População do Brasil por sexo e idade: 2000-2060. Disponível em:

https://ww2.ibge.gov.br/home/estatistica/populacao/projecao_da_populacao/2013/default.shtm. Acesso em: 15 jan. 2020.

IPEA-INSTITUTO DE PESQUISA E ECONÔMIA APLICADA.LOTTA, G.; FAVARETO, A. (Org.). Os Arranjos Institucionais dos Investimentos em Infraestrutura no Brasil: uma análise sobre seis grandes projetos do Programa de Aceleração de Crescimento, 2016. Disponível em: http://www.ipea.gov.br/portal/index.php?option=com_content\&view=article\&id=29014\&ltemid=406. Acesso em: 08 jan. 2020.

LEVY, B. R. et al.Ageism Amplifies Cost and Prevalence of Health Conditions. The Gerontologist, n. 11, p. 1-8, 2018.

MIRANDA, D. et al.Population aging in Brazil: current and future social challenges and consequences. 
Revista Brasileira de Geriatria e Gerontologia, v. 19, n. 3, p. 507-519, 2016.

SILVEIRA, H. R.; COSTA, R. E. D. OLIVEIRA. V. S. A descentralização de Políticas Públicas no

Brasil e o Sistema Único de Assistência Social. Journal of Chemical Information and Modeling, v. 12 Suppl 1, n. 9, p. 1-29, 2005.

VERAS, R. Envelhecimento populacional contemporâneo: demandas, desafios e inovações. Revista de Saúde Pública, v. 43, n. 3, p. 548-554, 2009.

VERAS, R. P.; OLIVEIRA, M. Envelhecer no Brasil: a construção de um modelo de cuidado. Ciência \& amp; Saúde Coletiva, v. 23, n. 5, p. 1929-1936, 2018.

WHO-WORLD HEARTH ORGANIZATION. Active Ageing: A Policy Framework. The Aging Male, v. 5, n. 1, p. 1-37, 2002. Disponível em:

http://www.informaworld.com/openurl?genre=article\&doi $=10.1080 / 713604647 \&$ magic $=$ crossref $\% 7 C \% 7$ CD404A21C5BB053405B1A640AFFD44AE3. Acesso em: 15 jan. 2020.

WHO-WORLD HEARTH ORGANIZATION. Healthy ageing. Disponível em:

http://www.who.int/ageing/WHO-ALC-10-priorities.pdf?ua=1. Acesso em: 25 mar. 2020.

WHO-WORLD HEARTH ORGANIZATION. What is "active ageing"?World Health Organisaion, 2014. Disponível em: https://www.who.int/ageing/healthy-ageing/en/. Acesso em: 10 jan. 2020.

WHO-WORLD HEARTH ORGANIZATION. World Report on Ageing and Health. Geneva, 2015.

Disponível em: https://www.who.int/ageing/publications/world-report-2015/en/. Acesso em: 17 jan. 2020.

WILLIG, M. H.; LENARDT, M. H.; MÉIER, M. J. A Trajetória Das Políticas Públicas Do Idoso No Brasil: Breve Análise. Cogitare Enfermagem, v. 17, n. 3, p. 574-577, 2012.

WONG, L. L. R.; CARVALHO, J. A. The rapid process of aging in Brazil: serious challenges for public policies.Revista Brasileira de Estudos de População, v. 23, n. 1, p. 5-26, 2006.

Notas

1 241: Assistência ao Idoso; 242: Assistência ao Portador de Deficiência; 243: Assistência à Criança e ao Adolescente; e, 244: Assistência Comunitária. 